\title{
Relaxation dynamics of a system with a grain boundary
}

\author{
D. B. Abraham, ${ }^{1,2}$ Ville Mustonen, ${ }^{1,2}$ and A. J. Wood ${ }^{1,3}$ \\ ${ }^{1}$ Theoretical Physics, University of Oxford, Oxford OX1 3NP, United Kingdom \\ ${ }^{2}$ Laboratory of Computational Engineering, Helsinki University of Technology, P.O. Box 9203, FIN-02015, Finland \\ ${ }^{3}$ School of Physics, James Clerk Maxwell Building, University of Edinburgh, Edinburgh, EH9 3JZ, United Kingdom
}

(Received 26 July 2004; published 27 December 2004)

\begin{abstract}
Recently an interesting phase transition scenario in the context of a planar Ising system with a grain boundary was revealed [Abraham et al., Phys. Rev. Lett. 93, 076101 (2004)]. In this paper we study in more detail the relaxation dynamics of this system and show how the grain boundary acts as a guide spatially confining matter transport. This is done by performing simulations using the continuous time Monte Carlo algorithm with Kawasaki dynamics. We also set up the problem as a solid-on-solid model and formulate corresponding Langevin equations for the dynamics in two cases, with and without matter conservation.
\end{abstract}

DOI: $10.1103 /$ PhysRevE.70.066138

PACS number(s): 05.70.Np, 68.08.Bc, 68.35.Rh

\section{INTRODUCTION}

Analytic approaches to equilibrium interfacial phase transition are now well developed within the context of statistical mechanics. Detailed and precise theories of the equilibrium properties of the delocalization or wetting transition have been developed in all dimensions [2,3]. In two dimensions, where an exact transfer matrix solution is possible via the Ising model [4], the transition is very well understood and we have a precise way of understanding the unbinding mechanism. The solid-on-solid (SOS) limit [5], where overhangs in the interfacial configuration are ignored, may also be considered. This approximation allows the interface location to be defined easily as a single valued function in the coordinate direction parallel to the wall. The problem can then also be formulated as a, albeit simpler, transfer problem which can then be critically compared to the exact Ising results $[4,6-8]$. In common with most areas of statistical mechanics, however, the understanding of nonequilibrium properties or dynamics within these systems, even in low dimensions, is less complete.

Simulation studies have made increasingly significant contributions to understanding the dynamics of systems exhibiting phase transitions. Especially in more recent years Monte Carlo and molecular dynamics simulations, combined with modern computing power, have enabled more realistic situations to be tackled [9-14].

An approach should be possible through the use of a Langevin equation [15], but this formalism cannot be well defined for a simple SOS model of an interface running next to a wall in two dimensions. The strict restriction $\ell>0$, where $\ell$ is the distance of the interface from the wall, is difficult, if not impossible, to implement without losing the Gaussian properties of the noise term. If the white noise term is left unaltered in the problem, as a "small" parameter in some sense, then the model cannot hope to sample from trajectories that move close to the wall. It is well established, in the equilibrium theory, that including excursions of the interface that closely approach the wall is crucial to correctly define physical parameters, i.e., contact angles and point tensions, see for example Ref. [16].

Despite this, some analytic progress has been made with this approach [17], but with a model defined using a horizon- tal solid-on-solid (HSOS) model [18] (i.e., ignoring overhangs so that the interface function is single valued in the coordinate direction perpendicular to the wall). This situation was adopted to model the immediate vicinity of the three phase contact point and thus to model the dynamics of the contact angle. The similar approach has also been used by Collet and co-workers [19], who consider a situation where the matter in the system is conserved.

In this paper we present a detailed analytic and numerical study on the relaxation dynamics of a system exhibiting the geodesic-to-zigzag (GZZ) transition. This continuous interfacial transition has been recently reported and an exact solution presented [1]. We will show how certain features of this exact solution combined with our Ising simulations strongly suggests that studying the problem within a horizontal solidon-solid approximation will give comparable results. Recent work confirmed this hypothesis in the equilibrium case [20]. Here we demonstrate how this HSOS formulation enables us to apply previous work $[17,19]$ to study the dynamics of the system with a grain boundary. We can then compare these HSOS results with the data garnered from MC simulations of the corresponding Ising system.

The paper is arranged as follows. In Sec. II we define the model to be studied. In Sec. III we present the details of our Ising simulations by examining both the static, equilibrium properties, and the dynamical, nonequilibrium ones. We are thus able to carefully cross check all our simulations with analytical results where available. In Sec. IV we introduce the appropriate solid-on-solid model for the static problem with two alternative approximations. In Sec. $\mathrm{V}$ we derive Langevin equations for the HSOS. We present a full analytic result for the matter nonconserving case and a similar result for the matter conserving case up to the level of computer algebra. Finally we study numerically, with simplistic analytical justification, the matter conserving case when the HSOS system is inhomogeneous. In this case we show that the conflicting energy balances induce a form of frustration. We then summarize our paper and findings in Sec. VI.

\section{MODEL AND THE “GZZ” TRANSITION}

Consider a planar Ising ferromagnet with nearestneighbor interactions and zero bulk magnetic field with a 
strip geometry and at a temperature $T$ below the bulk critical value $T_{c}$. By fixing the spin values to be all +1 on one edge and -1 on the other and taking the system size to be thermodynamically large we will have two oppositely magnetized bulk phases separated by a single interface with average orientation $(1,0)$. The positivity of the surface tension ensures that we have only a single such domain boundary in the system. Suppose additionally that the bonds in the $(0,1)$ direction between a single pair of adjacent rows of spins are weakened to form simple model of a grain boundary.

The investigation of models of this type has been the subject of much study in the wetting community for the last twenty years. It is now well understood that for a system described above with a central grain boundary the interface will be bound to the defect line for all temperatures $T<T_{c}$. If, in the thermodynamic limit, the boundary is a finite distance from one of the walls or lies next to one of the walls then a phase transition will occur at some temperature $T_{w}$, with $T_{c}>T_{w}>0$. This transition is identified as the well understood delocalization or wetting transition. An elegant argument, due to Fisher, described this by understanding the typical wandering of the interface from the defect line in terms of the first return times of a suitably chosen random walk. By utilizing the Boltzmann weights of the differing interfacial steps as the probability parameters in the stochastic process the mapping can be made explicitly [21].

Here we consider an interface crossing such a grain boundary, which is centrally positioned, at an angle implemented as in Fig. 1 by fixing spins at the edges with a given offset. As the width of the system diverges, the limit must be carefully taken to ensure that the angular boundary condition is preserved. When $b=1$ (no grain boundary) the optimal, most likely, path is the shortest, geodesic one. But suppose $0<b<1$ (ferromagnetic grain boundary); a zigzag path as in Fig. 1 allows the interface to profit energetically from intersecting the weakened bonds.

An analytic study of this phenomenon was presented recently as well as simulations of the relaxation dynamics of this system implemented using Kawasaki dynamics [1]. The exact solution for the phase boundary is

$$
b^{*}=\frac{1}{2 K_{1}} \ln \left(\frac{\tan \phi \cosh 2 K_{1}+\sqrt{1+\tan ^{2} \phi \sinh ^{2} 2 K_{1}}}{\tan \phi+1}\right)
$$

which is explicitly independent of the $K_{2}$ bond strength; an unexpected result. We shall utilize this observation later to motivate an approximation. As the phase boundary forms a plane in the three dimensional parameter space $(b, \phi, T)$ we may express it in several differing forms. The form (1) phase turns out to be algebraically convenient and thus determines the critical value of $b=b^{*}$ at which we may expect a transition for a given value of $\phi$ and $K_{1}(\propto 1 / T)$. Also note that this transition is not a necessary precursor transition as we approach bulk criticality; the system may be in either configuration as $T \rightarrow T_{c}$. The reader is referred to Fig. 2 for an example of three curves at a given, fixed angle in the $(b, t)$ plane ( $t$ is a reduced temperature).

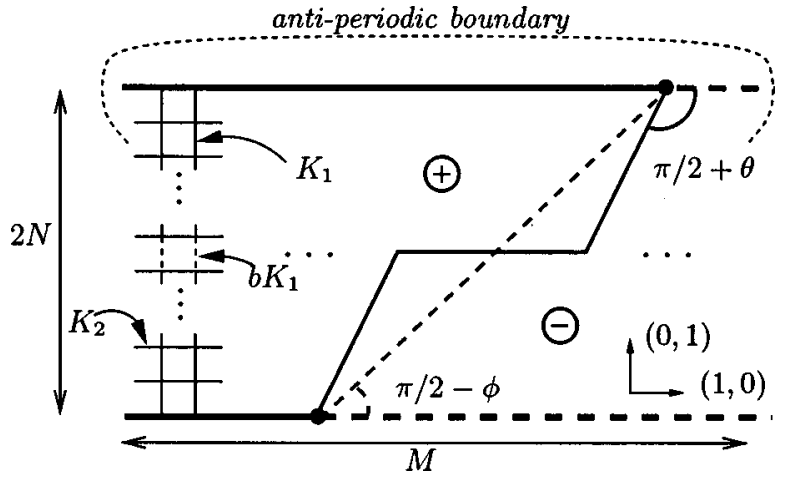

FIG. 1. A schematic picture of the simulated $(M \times 2 N)$ Ising system described in the text. The spins at the solid horizontal boundaries are fixed to be positive and the ones at the broken horizontal boundaries are fixed to be negative. Vertical bonds $K_{1}$ in the middle are weakened by factor $b$; this is the grain boundary. The dashed line depicts a mean macroscopic interface crossing the system at an angle $\phi$, the heavy solid line shows a typical zigzag interface configuration, where the middle section is pinned to the grain boundary. The transition studied, the GZZ, is the crossover between these two configurations. A perfect zigzag is formed when $\theta=0$, this corresponds to the zero temperature configuration for a system with a grain boundary $(b<1)$ and is also one of the degenerate ground states for a system without a grain boundary $(b=1)$. This configuration is the initial configuration for the relaxation process studied in this paper. The bond strengths of the model are included for reference, where $K_{j}=J_{j} / k_{B} T$, in all our simulations $K_{1}=K_{2}$. The circled plus (minus) sign depicts that the bulk is on average at plus (minus) magnetization on the left (right) hand side of the interface separating the phases.

Here we study the relaxation dynamics in more detail. In all cases the interface will relax from a perfect zigzag (see Fig. 1 with $\theta=0$ ) to zigzag configuration $\theta>0$. We show that when the zigzag configuration equilibrates in this manner, the dynamics of approach to equilibrium favors, in the main, motion of matter (in the lattice gas interpretation) along the line of defect bonds. This, as a generalization of what happens at equilibrium, may be termed dynamical confinement.

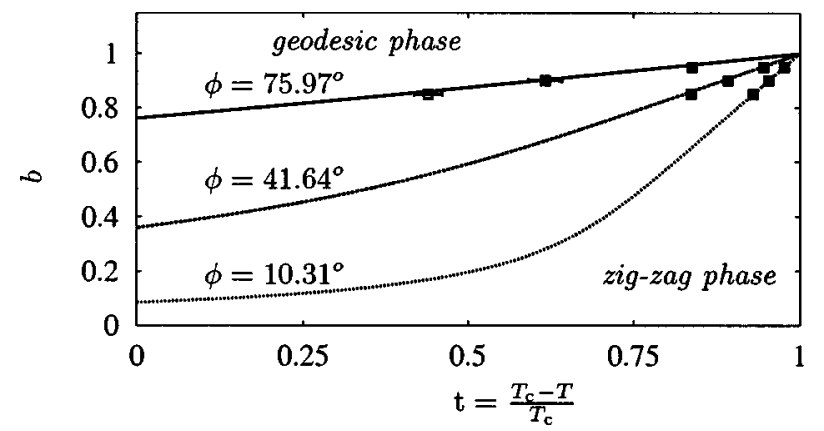

FIG. 2. Lines depict the phase diagram of the GZZ transition for three different angles calculated from the exact result (1). Phase transition points defined by MC for these angles are plotted for $b$ $=0.95,0.9,0.85$. The error bars are the standard deviations over ten realizations. Starting from point $(b=1, t=0)$ when the phase boundary for a given angle is being crossed geodesic interface configuration changes to a zigzag. 
It is the primary purpose of this paper to investigate this phenomenon and the relaxation dynamics further.

\section{ISING SIMULATIONS}

\section{A. Statics}

Before considering the dynamics of the model we studied the statics of the GZZ transition to gain understanding on the finite size effects, and to get information about the equilibrium state. This is necessary to ensure that our simulations with Kawasaki dynamics are performed at the right point of the phase diagram and that they indeed reach the correct equilibrium state.

Defining phase boundaries for the GZZ transition using conventional Monte Carlo methods would be a daunting task because one would be forced to do a number of simulations with different bond weakening factors $b$, angles $\phi$, and temperatures $T$. We could of course use the exact solution as a guide to limit our parameter space, and do the simulations only in the vicinity of the predicted transition line but this would not be an independent and unbiased test for the predictions. Instead we use $N$-fold implementation of the WangLandau sampling [22] introduced by Schulz et al. [23]. In Wang-Landau sampling, a random walk in energy space is performed to get accurate estimates for the energy density of states $g(E)$. The main advantage of the method is that $g(E)$ allows us to calculate information about thermodynamic quantities for all temperatures $T$ with a single simulation.

Our simulated system is depicted in Fig. 1 To create the interface we fix spins on the upper and lower boundaries; furthermore, an antiperiodic boundary condition is used in the $(1,0)$ direction. While performing the last few iterations of the energy random walks we also compute averages of magnetization and its moments in the microcanonical ensemble. After the random walks in energy space have converged and appropriate normalization has been done for the energy density of states, we can calculate canonical averages of magnetization,

$$
\langle|m|\rangle_{T}=\sum_{E}\langle|m|\rangle_{E} g(E) e^{-E / k_{B} T} / Z
$$

and its second moment

$$
\left\langle m^{2}\right\rangle_{T}=\sum_{E}\left\langle m^{2}\right\rangle_{E} g(E) e^{-E / k_{B} T} / Z,
$$

where $Z$ is the partition function. Now we can evaluate susceptibility $\chi(T)=N M / T\left(\left\langle m^{2}\right\rangle_{T}-\langle|m|\rangle_{T}^{2}\right)$ for all $T$ and define the GZZ transition points from the susceptibility peaks for different weakenings $b$ and angles $\phi$. We performed simulations described above for three different bond weakenings $b=0.95,0.9,0.85$ and for three angles $\phi=75.97^{\circ}, 41.64^{\circ}$, $10.31^{\circ}$ [corresponding lattices $(63 \times 14),(47 \times 46)$, and $(23$ $\times 78$ ), respectively]. Results are shown in Fig. 2. Finite size effects change the phase diagram obtained from MC simulations; we find that the transition temperature is lowered (see, for example Ref. [24]), but this correction is small and indeed with low temperatures becomes of the order of statistical error. Our exact analysis allow us to treat the finite size
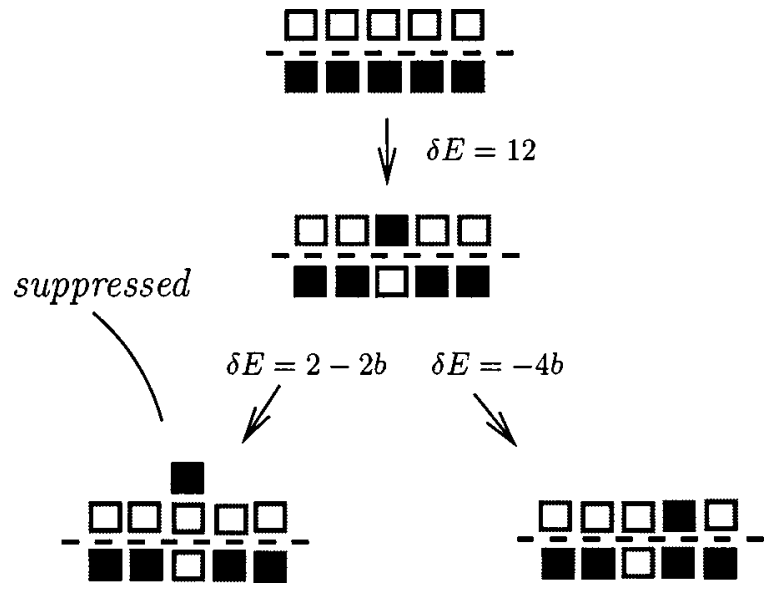

FIG. 3. A schematic picture showing the basic microscopic processes starting the relaxation process. The dashed horizontal lines depict the interface at the grain boundary. From the top, initially the interface is at the grain boundary, first possible move costs $\delta E$ $=12$. Then the created spin pair either recombines, dissociates along the grain, or evaporates to the spectator phase. Importantly this evaporation is suppressed by the energy cost $\delta E=2-2 b>0$ confining the matter transport in the grain boundary.

effects analytically and we find that the corrections become significant only when the system approaches the bulk critical point [25].

One conclusion of the simulations for the statics is that the phase diagram is virtually untouched by the finite size effects; this is true for low temperatures. In the next section we operate only in this region of the phase diagram.

\section{B. Kawasaki dynamics}

In studying out-of-equilibrium properties of systems one usually focuses on one of two things; one can either study systems with driving forces which prevent them to reach equilibrium [26], or the relaxation of systems from an initial state to an equilibrium configuration at a given temperature. In the context of an Ising system with a grain boundary, keeping in mind the lattice gas interpretation, a very interesting question is how the matter is transported in thermalizing from the $T=0$ configuration as an initial configuration (interface in a zigzag configuration with $\theta=0$ in Fig. 1) to equilibrium at $T>0$. The standard chemical physical idea is that the most important paths are those involving the least excitation energy. This picture is complicated here because there are many such paths in principle, furthermore diffusion through the bulk phase as a relaxation mechanism cannot be ruled out without a careful analysis. What we show here is that matter flows along the grain boundary, by creating spin flip pairs aligned on $(0,1)$, which dissociate and then diffuse freely along either side of the boundary, except for occasional trapping or collision; see Fig. 3. They are then absorbed in the corners formed by the junctions of the vertical interface sections and the interface section pinned at the grain boundary, promoting relaxation to the equilibrium wedge angle.

The fundamental difficulty of performing simulations of relaxation processes is that the choice of dynamics changes 

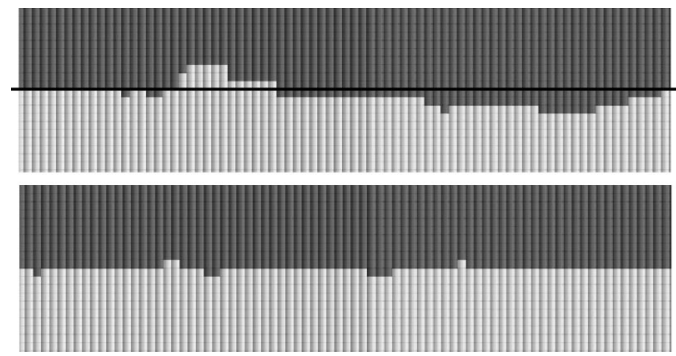

FIG. 4. The qualitative picture. Typical snapshots of the simulated Ising systems showing the middle section of the interface early on the relaxation process. The system above does not have a grain boundary $(b=1)$ and system below has one $(b=0.85)$. The snapshots give qualitative confirmation of the capillary fluctuations [the middle line is explicitly depicted for case $(b=1)$ ] as the relaxation mechanism for the system without a grain boundary and also show the confined matter transport happening at the grain boundary when there is one.

the rates and the way the systems equilibrate. We want the dynamics to capture two features of a realistic physical system, first, matter must be conserved and second, the dynamics must be local. Using Kawasaki dynamics with local updates fulfills these requirements. In order to make simulation efficient at low temperatures, we implemented the Kawasaki dynamics with the continuous time algorithm [27]. The Ising system is set up as in the static case. We considered two systems, one without a grain boundary $(b=1.0)$ and one with $(b=0.85)$. The lattice size was $M=139,2 N=34, \phi=75.97^{\circ}$ and temperature in scaled units $\mathrm{t}=\left(T_{c}-T\right) / T_{c}=0.65$. The system with the grain boundary will relax to a zigzag configuration since the field $b<b_{c}$ for the given parameters; see the phase diagram in Fig. 2.

In Fig. 4 typical snapshots of the simulated interfaces are shown and in the following we make the observed confined matter transport phenomenon quantitative. The main interest of the matter transfer mechanism is the movement in the $(1,0)$ direction. Accordingly, we define the total matter transfer for each row of spins as

$$
\mathcal{S}_{j}^{b}\left(t_{\mathrm{MC}}\right)=\mathcal{S}_{j}^{b}\left(t_{\mathrm{MC}}-1\right)+\sum_{i} f\left(\sigma_{i, j}, \sigma_{i+1, j} ; t_{\mathrm{MC}}\right) \sigma_{i+1, j},
$$

where $t_{\mathrm{MC}}$ is the number of MC steps, $f=1$ if the spin pair $\sigma_{i, j}, \sigma_{i+1, j}$ is flipped between $t_{\mathrm{MC}}-1$ and $t_{\mathrm{MC}}$ time steps, otherwise $f=0$ and $b$ the bond weakening in the middle.

In Figs. 5 and 6 the time evolutions of total matter transfer for each row of spins are plotted for $b=1$ and $b=0.85$, respectively. It can be clearly seen that whereas the sum of total matter transfers for the case $b=1$ gets contributions from several rows near the central line, in the case of $b$ $=0.85$ the main contribution comes only from the middle pair touching the grain. To make this observation clear we calculate the normalized average total matter transfer $\mathcal{I}_{j}^{b}$ $=\left\langle\mathcal{S}_{j}^{b}\right\rangle / \Sigma_{j}\left\langle\mathcal{S}_{j}^{b}\right\rangle$. Looking at these fractions for the $b=1$ case, we find that the middle two rows contribute less than quarter of the total, i.e., $\mathcal{I}_{17}^{1}+\mathcal{I}_{18}^{1}=0.23$, the next ones $\mathcal{I}_{16}^{1}+\mathcal{I}_{19}^{1}$ $=0.14, \mathcal{I}_{15}^{1}+\mathcal{I}_{20}^{1}=0.09, \mathcal{I}_{14}^{1}+\mathcal{I}_{21}^{1}=0.06$, and all the rest $\sim 0.02$ each, this is diffusion through the bulk phase.

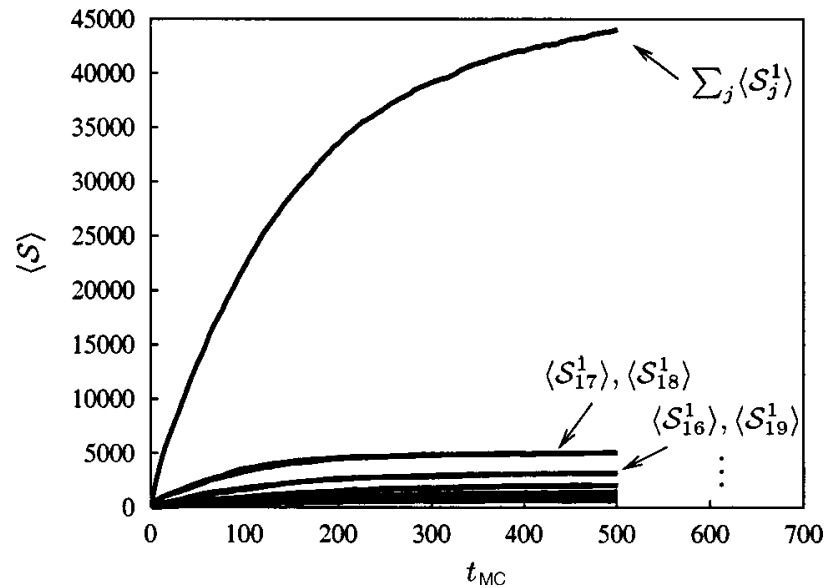

FIG. 5. The quantitative picture for a system without a grain boundary $(b=1)$. The time development of total matter transfer for each row of spins as a function of time $t_{\mathrm{MC}}$. The matter transfer is divided between the rows close to center, the relative importance gradually lowering as the distance from the middle increases, confirming that the system relaxes using capillary fluctuations. Lines are averages over $450 \mathrm{MC}$ realizations, time units are arbitrary.

Consider now the system with a grain boundary ( $b$ $=0.85$ ). The two middle rows, i.e., the grain boundary, contribute more than two-thirds of the total, $\mathcal{I}_{17}^{0.85}+\mathcal{I}_{18}^{0.85}=0.68$, and the rest of the rows each contribute $\sim 0.02$. Comparing these fractions leads to the following conclusions. While the system without a grain boundary equilibrates using capillary fluctuations, the case with the grain boundary will relax using an alternative mechanism; the section of the interface pinned to the grain boundary will act as a diffusive guide for the particles. It is also important to notice that in the case $b=1$ the amount of total matter needed to transfer before the system reaches equilibrium is greater, $\Sigma_{j}\left\langle\mathcal{S}_{j}^{1}\right\rangle / \Sigma_{j}\left\langle\mathcal{S}_{j}^{0.85}\right\rangle$

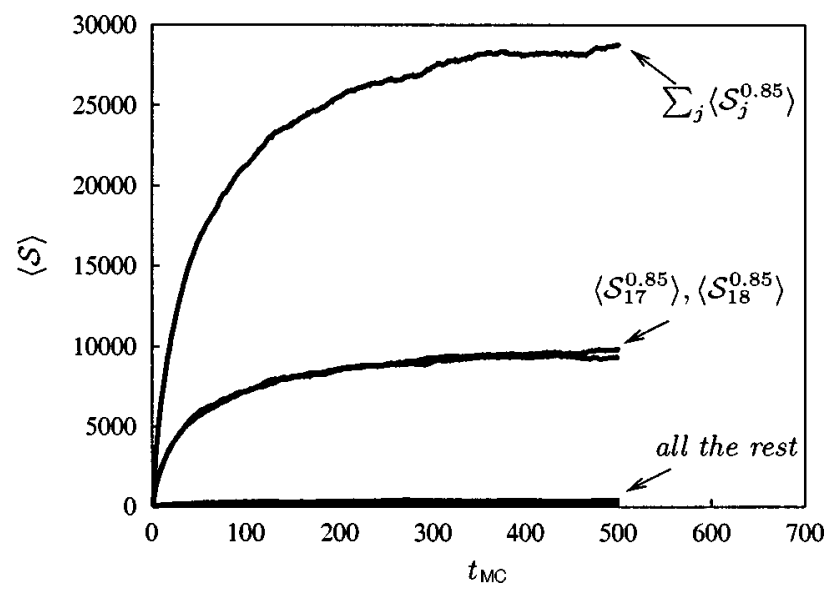

FIG. 6. The quantitative picture for a system with a grain boundary $(b=0.85)$. The time development of total matter transfer for each row of spins as a function of time $t_{\mathrm{MC}}$. The middle two rows touching the grain dominate the matter transfer process, contributing together $68 \%$ of the total, whereas all the rest contribute less than $2 \%$ each. This confirms the picture of spatially confined matter transport. Lines are averages over $450 \mathrm{MC}$ realizations, time units are arbitrary. 


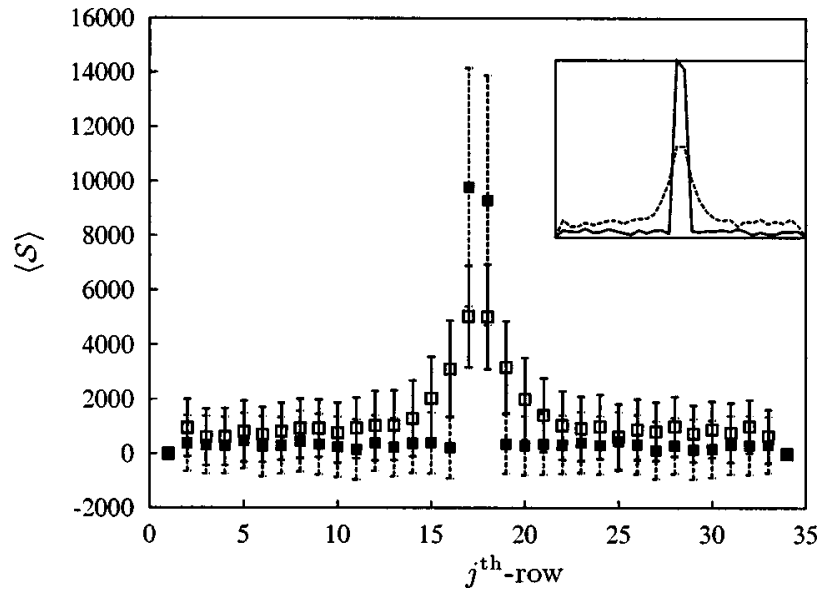

FIG. 7. The non-normalized average total matter transfer as a function of row number $j$ after the systems have equilibrated. Black squares are for the the system with a grain boundary and empty squares for the system without one. The error bars are standard deviations defined over $450 \mathrm{MC}$ realizations. Inset: the same data but with connected points and error bars left out. The solid line corresponds to a system with a grain boundary and the dotted line to one without. These transport "spectra" demonstrate that also the absolute number of particles going through the middle two rows is increased when the grain is introduced even though the system without a grain boundary needs to move more matter to reach equilibrium.

$=1.47$, but still $\left(\left\langle\mathcal{S}_{17}^{1}\right\rangle+\left\langle\mathcal{S}_{18}^{1}\right\rangle\right) /\left(\left\langle\mathcal{S}_{17}^{0.85}\right\rangle+\left\langle\mathcal{S}_{18}^{0.85}\right\rangle\right)=0.51$, meaning that the absolute number of particles using the middle two rows is doubled when the grain boundary is introduced; see Fig. 7. This means that by introducing a defect line in the system one confines spatially the bulk of the matter transport to the minimum energy pathways.

The conclusions made above, combined with the striking fact that the phase boundary for the GZZ transition was found to be independent of the $K_{2}$ bond strengths, suggest strongly that we should study the system with a solid-onsolid approximation.

\section{SOLID-ON-SOLID MODEL FOR GZZ SYSTEM}

We set up a horizontal solid-on-solid (HSOS) model [18] to study the relaxation dynamics of the system with a grain boundary and an interface crossing it at an angle $\phi$; see Fig. 8. Following the logic where one needs to understand statics before trying to understand dynamics, we first solve (or quote the results) of the static case and then derive Langevin dynamics for the model in the next section.

The Hamiltonian for an HSOS model can be written as

$$
\mathcal{F}\left(h_{1}, \ldots, h_{2 N}\right)=\sum_{j=1}^{2 N} u\left(h_{j}-h_{j-1}\right),
$$

where the function $u$ is chosen to be

$$
u\left(h_{j}-h_{j-1}\right)=\tau \sqrt{1+\left(h_{j}-h_{j-1}\right)^{2}} .
$$

This generic description for an HSOS model is derived by applying the Pythagoras theorem to the exposed length of an

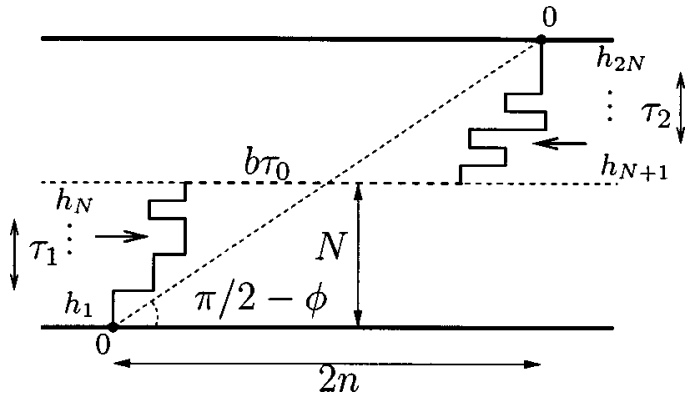

FIG. 8. HSOS setup for studying the GZZ transition and the relaxation dynamics of the model. The system has $2 N$ rows and fixed boundary conditions. In the upper half system the couplings between HSOS variables are $\tau_{2}$ and in the lower half $\tau_{1}$. The grain boundary is modeled by modified coupling $b \tau_{0}$ between the $h_{N}$ and $h_{N+1}$. The angle $\phi$ is defined similarly to the Ising system. The boundary conditions force the interface with $b \tau_{0}=\tau_{1}=\tau_{2}$ to cross the system on average at an angle $\phi$ whereas a symmetric zigzag configuration is formed if $b \tau_{0}<\tau_{1}=\tau_{2}$.

interface section connecting neighboring columns. Equation (5) should be interpreted mesoscopically; the units do not necessarily correspond to atomic lengths. Instead we may consider $h_{j}$ 's as continuous variables.

We have studied two approximations of Eq. (5) in the context of the GZZ transition:

$$
\mathcal{F}=\sum_{j=1}^{N} \tau_{1}\left|h_{j}-h_{j-1}\right|+b \tau_{0}\left|2 n-h_{N+1}-h_{N}\right|+\sum_{j=N+2}^{2 N} \tau_{2}\left|h_{j}-h_{j-1}\right|
$$

and the Gaussian case,

$$
\begin{aligned}
\mathcal{F}= & \sum_{j=1}^{N} \tau_{1}\left(h_{j}-h_{j-1}\right)^{2}+b \tau_{0}\left|2 n-h_{N+1}-h_{N}\right| \\
& +\sum_{j=N+2}^{2 N} \tau_{2}\left(h_{j}-h_{j-1}\right)^{2} .
\end{aligned}
$$

Equations (7) and (8) represent the two possible approximations of the interfacial free energy function defined in Eq. (6). Essentially, for $h_{j}-h_{j-1}$ large, $u(x) \sim \tau|x|$ but for $h_{j}$ $-h_{j-1}$ small it is more appropriate to perform the usual binomial expansion on Eq. (6) to find the familiar square gradient term (and a constant which is removed by normalization). It is clear that in both cases the former, modulus approximation will be the most appropriate for the term considering the defect line. However, the choice is not so clear for the remaining terms. For large angles, we may expect Eq. (7) to be the most appropriate. For small angles, or at small times (in the relaxation from the perfect zigzig sense), then the Gaussian terms, Eq. (8), should be a better approximation. In other words, keeping in mind the physical picture established by studying both dynamics and statics of the full Ising description of the problem, we need to resort to the Gaussian form of the interaction between columns other than the middle two as they show long wavelength, i.e., capillary fluctuations. However, as mentioned already, the modulus approximation 
needs to be chosen for the columns touching the grain boundary. The $2 n$ term in the energy connecting the two middle columns in Eqs. (7) and (8) is a result of a mapping $h_{j} \rightarrow 2 n-h_{j}$ for the columns $j>N+1$. This mapping will make the initial condition for our Langevin study equal to zero and in what follows the derived equations will appear in more concise form.

In Ref. [20] we studied the equilibrium properties of Eq. (7) and the exact Ising result for the phase boundary was recovered in an appropriate scaling limit. We have also done similar calculation for Eq. (8) and for completeness the main results of this calculation are presented in the Appendix. Note that Eq. (8) explicitly treats the central column separately and thus fails to recover the expected behavior in the limit $b \rightarrow 1$. Our results reflect this.

The incentive for doing the HSOS calculations in the first place is the relative ease of generalizing the system to be inhomogeneous, i.e., the couplings $\tau_{j}$ are different below and above the grain boundary. Furthermore, the HSOS formulation, with the Gaussian approximation (8), allows us to study the early time relaxation dynamics of the system analytically.

\section{LANGEVIN DYNAMICS OF THE HSOS MODEL}

In this section we formulate Langevin dynamics for the studied system (see Fig. 8) and solve the following equations within the Gaussian approximation (8). The dynamics are presented both for a matter nonconserving system and for a matter conserving system.

The dynamics of a time-dependent interface can be written in terms of the Langevin equation $[15,28,29]$,

$$
\frac{\partial h_{j}}{\partial t}=-\frac{\lambda}{2} \frac{\partial \mathcal{F}}{\partial h_{j}}+\eta_{j}(t),
$$

where the white noise $\eta(t)$ is defined by

$$
\left\langle\eta_{j}(t)\right\rangle=0, \quad\left\langle\eta_{j}(t) \eta_{j^{\prime}}\left(t^{\prime}\right)\right\rangle=\Omega \delta_{j, j^{\prime}} \delta\left(t-t^{\prime}\right) .
$$

In the following the arbitrary time scale $\lambda^{-1}$ and noise amplitude $\Omega$ are set to 1 .

As we observed in the preceding section, the Gaussian approximation used subsequently is valid for either small times or small angles. The reader should be aware of this limitation when examining our results. Certainly Eq. (8) is a very poor approximation as the system approaches the geodesic configuration. Ideally we would like to model the complete relaxation process, from perfect zigzag $(\theta=0)$ to this point $(\theta=\phi)$, by understanding the crossover from Eq. (8) to Eq. (7), but this scheme is beyond the scope of the present paper.

\section{A. Matter nonconserving dynamics}

Using Eq. (9) directly with Eq. (8) leads to the following set of coupled differential equations:

$$
d \mathbf{h}=-\left(A_{x} \mathbf{h}+\frac{b \tau_{0}}{2}\left(\mathbf{e}_{N+1}+\mathbf{e}_{N}\right)\right) d t+d \eta,
$$

where $\mathbf{e}_{j}$ is a unit vector in a $2 N$ space with $j$ th element being 1 ,

$$
\begin{gathered}
A_{x}=\left(\begin{array}{cccc}
\tau_{1} A_{1} & 0 \\
0 & \tau_{2} A_{2}
\end{array}\right), \\
A_{1}=\left(\begin{array}{ccccccc}
2 & -1 & 0 & 0 & 0 & \ldots & \ldots \\
-1 & 2 & -1 & 0 & 0 & \ldots & \ldots \\
0 & -1 & 2 & -1 & 0 & \ldots & \ldots \\
& & & \ddots & & & \\
\ldots & \ldots & 0 & 0 & -1 & 2 & -1 \\
\ldots & \ldots & 0 & 0 & 0 & -1 & 1
\end{array}\right),
\end{gathered}
$$

and

$$
A_{2}=\left(\begin{array}{ccccccc}
1 & -1 & 0 & 0 & 0 & \ldots & \ldots \\
-1 & 2 & -1 & 0 & 0 & \ldots & \ldots \\
0 & -1 & 2 & -1 & 0 & \ldots & \ldots \\
& & & \ddots & & & \\
& & & & \ddots & & \\
\ldots & \ldots & 0 & 0 & -1 & 2 & -1 \\
\ldots & \ldots & 0 & 0 & 0 & -1 & 2
\end{array}\right) .
$$

We diagonalized matrix $A_{x}$ to solve Eq. (11), eigenvalues are

$$
\lambda_{q}=2 \tau_{1}\left(1-\cos \Theta_{q}\right), \quad \Theta_{q}=\frac{(2 q+1) \pi}{2 N+1}
$$

and

$$
\lambda_{q}=2 \tau_{2}\left(1-\cos \Theta_{q}\right), \quad \Theta_{q}=\frac{(2 q+1) \pi}{2 N+1}
$$

with $q=0,1, \ldots, N-1$. The first set of eigenvalues have associated normalized eigenvectors with components

$$
S_{j}^{(q)}=C \sin \left(j \Theta_{q}\right),
$$

where $C=2 / \sqrt{2 N+1}$, when $1 \leqslant j \leqslant N$ and $C=0$, for $N+1$ $\leqslant j \leqslant 2 N$. Similarly for the second set of eigenvalues,

$$
S_{j}^{(q)}=D \cos \left[(j-N-1 / 2) \Theta_{q}\right],
$$

where $D=2 / \sqrt{2 N+1}$, when $N+1 \leqslant j \leqslant 2 N$ and $D=0$, for 1 $\leqslant j \leqslant N$. After the orthogonal transformation Eq. (11) reads

$$
\frac{\partial \hat{h}_{q}(t)}{\partial t}=-\left(\tau_{1}+\tau_{2}\right) \lambda_{q}(t)+\frac{1}{2} b \tau_{0}\left(S_{N+1}^{(q)}+S_{N}^{(q)}\right)+\hat{\eta}_{q}
$$

in the component form, where $h_{j}(t)=\Sigma_{q} \hat{h}_{q}(t) S_{j}^{(q)}$ and $\eta_{j}(t)$ $=\Sigma_{q} \hat{\eta}_{q}(t) S_{j}^{(q)}$. Our definition of $\mathbf{h}$ makes the initial condition $\mathbf{h}(0)=\mathbf{0}$ for the perfect zigzag.

We can now solve the differential equation (19) resulting in

$$
\begin{aligned}
\hat{h}_{q}(t)= & \frac{b \tau_{0}}{2} \frac{1-e^{-\left(\tau_{1}+\tau_{2}\right) \lambda_{q} t}}{\left(\tau_{1}+\tau_{2}\right) \lambda_{q}}\left(S_{N+1}^{(q)}+S_{N}^{(q)}\right) \\
& +\int_{0}^{t} e^{-\left(\tau_{1}+\tau_{2}\right) \lambda_{q}(t-s)} \hat{\eta}(s) d s
\end{aligned}
$$

and transform $\hat{h}_{q}(t)$ back to the original basis, 


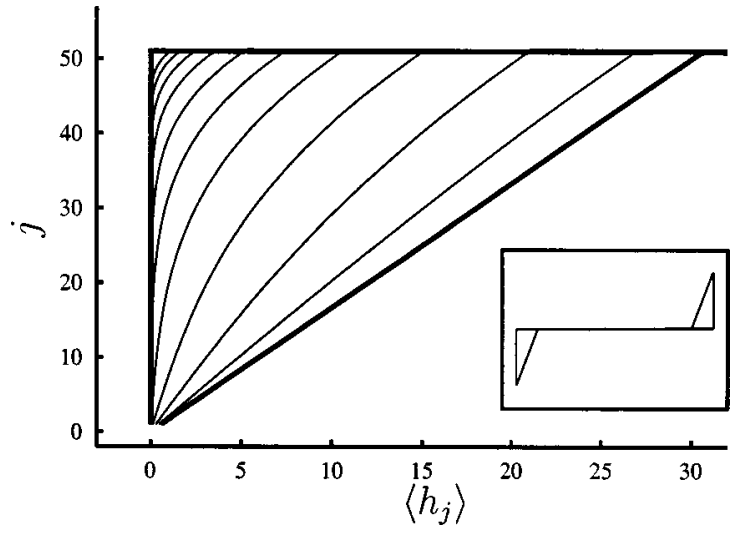

FIG. 9. The relaxation without matter conservation from a perfect zigzag configuration to a zigzag using HSOS model with Gaussian dynamics defined by Eq. (11). A set of interfaces at intermediate times have been depicted with thinner lines; only half the system is shown. In the inset the full system is depicted showing only the initial and equilibrium configurations.

$$
\begin{aligned}
h_{j}(t)= & \sum_{q}\left(\frac{b \tau_{0}}{2} \frac{1-e^{-\left(\tau_{1}+\tau_{2}\right) \lambda_{q} t}}{\left(\tau_{1}+\tau_{2}\right) \lambda_{q}}\left(S_{N+1}^{(q)}+S_{N}^{(q)}\right) S_{j}^{(q)}\right. \\
& \left.+\int_{0}^{t} e^{-\left(\tau_{1}+\tau_{2}\right) \lambda_{q}(t-s)} \hat{\eta}_{q}(s) S_{j}^{(q)} d s\right) .
\end{aligned}
$$

We considered $\left\langle h_{j}(t)\right\rangle$ and the variance $\left\langle h_{j}(t)^{2}\right\rangle-\left\langle h_{j}(t)\right\rangle^{2}$ resulting in

$$
\left\langle h_{j}(t)\right\rangle=\sum_{q} \frac{b \tau_{0}}{2} \frac{1-e^{-\left(\tau_{1}+\tau_{2}\right) \lambda_{q} t}}{\left(\tau_{1}+\tau_{2}\right) \lambda_{q}}\left(S_{N+1}^{(q)}+S_{N}^{(q)}\right) S_{j}^{(q)}
$$

and

$$
\left\langle h_{j}(t)^{2}\right\rangle-\left\langle h_{j}(t)\right\rangle^{2}=\sum_{q} \frac{1-e^{-2\left(\tau_{1}-\tau_{2}\right) \lambda_{q} t}}{2\left(\tau_{1}+\tau_{2}\right) \lambda_{q}}\left(S_{j}^{(q)}\right)^{2} .
$$

Langevin equations for a precursor film near a wall which favors spreading were derived and solved in Ref. [17]. Equations (22) and (23) are analogous to the spreading equations. After carefully replacing the summations by integrals, the main results state that the relaxation time scale is $\sim t^{1 / 2}$ and the amplitude of the fluctuations is of the order $\sim t^{1 / 4}$. In Fig. 9 the initial and equilibrium configurations of the system are depicted with intermediate steps of the relaxation.

\section{B. Matter conserving dynamics}

In order to derive the appropriate Langevin equation for conserved order parameter, we first consider a general Langevin equation with cross coupling of the noise and an arbitrary force $\mathbf{F}(\{\mathbf{h}\})$ term,

$$
\frac{\partial h_{i}(t)}{\partial t}=F_{i}(\{\mathbf{h}(t)\})+\left(\frac{2}{\beta}\right)^{1 / 2} \sum_{j=1}^{2 N} \sigma_{i j} \eta_{i}(t),
$$

where $\eta_{i}(t)$ are Gaussian noise terms defined through their first and second moments as in Eq. (10).

From such an equation we may derive a Fokker-Planck equation. From this form we will be able to infer the condi- tions on $\mathbf{F}(\{\mathbf{h}\})$ and $\sigma_{i j}$ that are required in order to achieve the Gibbs distribution with matter conservation in equilibrium. In their paper Collet et al. [19] use Ito calculus to perform this conversion. Here we use a less rigorous approach, and follow the derivation presented in ZinnJustin [30].

First we define a probability as

$$
\mathcal{P}(\{q\}, t)=\langle\delta(h(t)-q)\rangle,
$$

where here and subsequently the angle brackets denote an average over the noise and the $\delta$ function is a product over all the individual variables $h_{i}$ and $q_{i}$. We take a time derivative and make use of the chain rule to derive

$$
\frac{\partial \mathcal{P}(\{q\}, t)}{\partial t}=\left\langle\sum_{j} \frac{\partial h_{j}(t)}{\partial t} \frac{\delta}{\delta h_{j}(t)} \delta(\mathbf{h}(\mathbf{t})-\mathbf{q})\right\rangle .
$$

We may now use the symmetry of the delta function to write the functional derivative (denoted $\delta$ ) as a negative coordinate derivative (denoted $\partial$ ). We also use the Langevin equation (24) to derive

$$
\begin{aligned}
\frac{\partial \mathcal{P}(\{q\}, t)}{\partial t}= & -\sum_{j} \frac{\partial}{\partial q_{j}} F_{j}(\{q\}) \mathcal{P}(\{q\}, t) \\
& -\sum_{j}\left(\frac{2}{\beta}\right)^{1 / 2} \frac{\partial}{\partial q_{j}}\left\langle\sum_{k} \sigma_{j k} \eta_{k}(t) \delta(\mathbf{h}(\mathbf{t})-\mathbf{q})\right\rangle .
\end{aligned}
$$

We now follow Zinn-Justin closely to compute the second term. First, that given an arbitrary function of the Gaussian noise, $G(\eta(t))$, then

$$
\left\langle G(\eta(t)) \eta_{i}(t)\right\rangle=\Omega\left\langle\frac{\delta G(\eta(t))}{\delta \eta_{i}(t)}\right\rangle
$$

and second that the equal time functional derivative of the height function with respect to the noise is given by

$$
\frac{\delta h_{i}(t)}{\delta \eta_{j}(t)}=\frac{1}{2}\left(\frac{2}{\beta}\right)^{1 / 2} \sigma_{i j}
$$

By choosing $G(\eta(t))=\sigma_{j k} \delta(\mathbf{h}(\mathbf{t})-\mathbf{q})$ and setting the bookkeeping variable $\Omega$ to unity we may derive the FokkerPlanck equation derived in Collet et al., namely

$$
\begin{aligned}
\frac{\partial \mathcal{P}(\{q\}, t)}{\partial t}= & -\sum_{j} \frac{\partial}{\partial q_{j}} F_{j}(\{q\}) \mathcal{P}(\{q\}, t) \\
& -\frac{1}{\beta} \sum_{i, j, k} \frac{\partial^{2}}{\partial q_{i} \partial q_{j}} \sigma_{i k} \sigma_{j k} \mathcal{P}(\{q\}, t) .
\end{aligned}
$$

To impose the condition on the Gibbs distribution with matter conservation to be the equilibrium for Eq. (30), we choose

$$
F_{i}(\{\mathbf{h}\})=-\sum_{j, k=1}^{2 N} \sigma_{i k} \sigma_{j k} \frac{\partial \mathcal{F}(\mathbf{h})}{\partial h_{j}} .
$$

The conservation of matter in Eq. (24) is then a consequence of a conservative noise, 


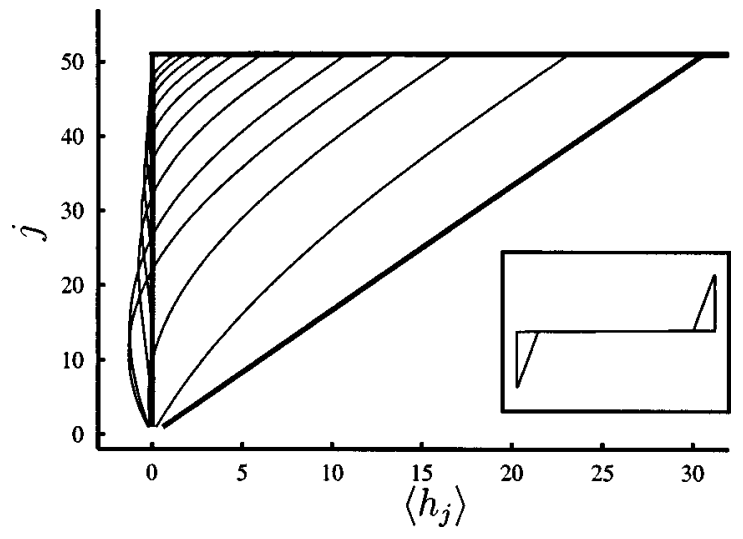

FIG. 10. The relaxation with matter conservation from a perfect zigzag configuration to a zigzag using the HSOS model with Gaussian dynamics defined by Eq. (34). A set of interfaces at intermediate times has been depicted with thinner lines; only half the system is shown. It can be seen that the imposed matter conservation introduces oscillations to move matter in the relaxation process in clear contrast to a system without conservation. In the inset the full system is depicted showing only the initial and equilibrium configurations; they coincide with the corresponding configurations without matter conservation.

$$
\sum_{j=1}^{2 N} \sigma_{j k}=0, \quad \forall k \in\{1, \ldots, 2 N\} .
$$

Remembering the Kawasaki dynamics with local spin exchanges used earlier in this paper, a natural choice for $\sigma$ is a local one [19],

$$
\sigma=\left(\begin{array}{cccc}
1 & & & \\
-1 & \ddots & & \\
& \ddots & 1 & 0 \\
& & -1 & 0
\end{array}\right)
$$

Putting it all together, the Langevin equation for the matter conserving dynamics reads

$$
d \mathbf{h}=\sigma \sigma^{\dagger}\left(-A_{x} \mathbf{h}+\frac{b \tau_{0}}{2}\left(\mathbf{e}_{N+1}+\mathbf{e}_{N}\right)\right) d t+\boldsymbol{\sigma} d \boldsymbol{\eta} .
$$

We have not been able to diagonalize the $\sigma \sigma^{\dagger} A_{x}$ matrix exactly, however, computing eigenvectors and eigenvalues numerically for the matrix is trivial and here we state only the results of Eq. (34). Imposing the matter conservation changes the relaxation process and even the equilibrium, as will be shown later. In Ref. [19] Collet et al. solved a system with free boundary conditions exactly and showed that the relaxation time scale is $\sim t^{1 / 4}$, and furthermore the average interface configuration shows oscillations with mean amplitude of oscillations scaling as $\sim t^{1 / 8}$. Our numerical solution for a system with fixed boundaries and a grain in the middle shows similar behavior. In Fig. 10 we depict a relaxation of a homogeneous system with conservation.

The next thing to do is to compare the HSOS results with matter conserving Langevin dynamics presented above to the Ising simulations with Kawasaki dynamics studied in Sec.
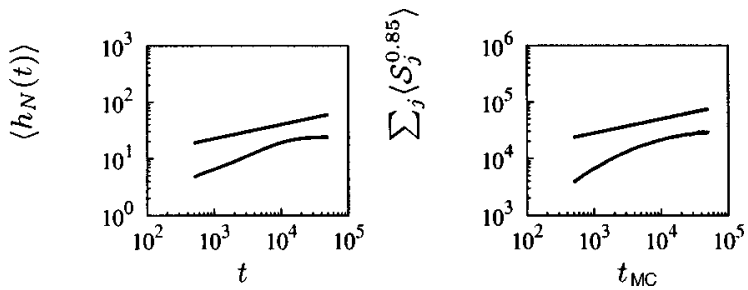

FIG. 11. The relaxation time scales for the HSOS model, from Eq. (34) (on the left) and for the Ising model studied in Sec. III (on the right). Both figures show also $\sim t^{1 / 4}$ curves on top of the numerical ones for comparison. The size of the HSOS system was chosen to be $N=16, n=66$ corresponding to our Ising simulations; see Fig. 8.

III. The comparison cannot be done directly as there is an obvious problem of fixing the time units and more importantly the noisiness of the Ising simulations prevented us from getting information about the exact interface coordinates as a function of time. However, the situation is not hopeless as we can use the total matter transfer (see Fig. 6) as a measure of the relaxation for the Ising system. The measure of relaxation from the HSOS system is simply the coordinate of the middle row $\left\langle h_{N}(t)\right\rangle$. In Fig. 11 the respective relaxation processes have been depicted, also $\sim t^{1 / 4}$ curves are shown for comparison. Both models show similar behavior with a fast start for the relaxation process then reaching $\sim t^{1 / 4}$ region followed by a final saturation as the system reaches the equilibrium.

\section{Inhomogeneous problem}

Before concluding, we briefly show some interesting observations and results for the inhomogeneous problem; consider $\tau_{1}>\tau_{2}>b \tau_{0}$. Whereas in the symmetrical case $\tau_{1}=\tau_{2}$ both systems with and without matter conservation relax to the same equilibrium configuration, when the system is inhomogeneous the equilibrium shapes are different. As we have not done the exact statics of the HSOS with conservation we show here a simple variational calculation verifying our numerical findings for Eq. (34) in the context of an inhomogeneous system.

First define the interfacial energy for curves $f$ and $g$ with a matter conservation constraint and free end points; see Fig. 12. We use here a square gradient approximation for simplicity,

$$
\begin{aligned}
E[f, g]= & \int_{0}^{N}\left\{\tau_{1} f^{\prime 2}+\tau_{2 g}^{\prime 2}+\lambda(f-g)\right\} d x \\
& +b \tau_{0}[2 n-f(N)-g(N)] .
\end{aligned}
$$

The variational argument thus reads

$$
\begin{aligned}
E[f+ & \delta f, g+\delta g]-E[f, g] \\
= & \delta f(N)\left\{\left.\tau_{1} \frac{\partial f}{\partial x}\right|_{N}-b \tau_{0}\right\}+\delta g(N)\left\{\left.\tau_{2} \frac{\partial g}{\partial x}\right|_{N}-b \tau_{0}\right\} \\
& -\int_{0}^{N} \delta f\left\{f^{\prime \prime}-\lambda\right\}+\int_{0}^{N} \delta g\left\{g^{\prime \prime}-\lambda\right\}
\end{aligned}
$$

and leads to 


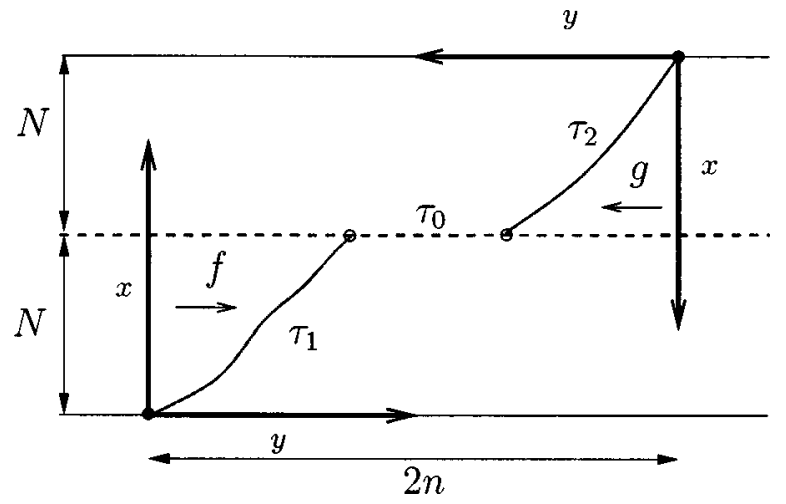

FIG. 12. Variational formulation for the inhomogeneous problem. Curves $f$ and $g$ have fixed points at the respective origins and free end points at the grain. The shape of curves and the end points at the grain come out from the calculation.

$$
\begin{gathered}
f(x)=\lambda x^{2}+A x+B, \\
g(x)=-\lambda x^{2}+C x+D .
\end{gathered}
$$

The variational parameters can be solved from the boundary conditions and the matter conservation $\int f d x=\int g d x$ resulting in

$$
\lambda=\frac{3 b \tau_{0}\left(\tau_{1}-\tau_{2}\right)}{8 N \tau_{1} \tau_{2}},
$$

$A=b \tau_{0} / \tau_{1}-2 \lambda N, B=0, C=b \tau_{0} / \tau_{2}+2 \lambda N$, and $D=0$. Hence in the case $\tau_{1}=\tau_{2}$ the equilibrium is constructed from straight line segments as expected. The key point in this simplistic approach is the emergence of opposite curvatures in the two interface sections (37). This is very natural occurrence, with a clear physical mechanism. Once the straight line sections are forced to a position beyond their grand canonical equilibrium, the new energy balance is between the bending terms and the weakening effect of the grain boundary. The interfaces will thus bend in such a way as to maximize the length of the section which is pinned to the grain boundary.

In Fig. 13 inhomogeneous systems are depicted and the bending of the equilibrium interfaces in the case of matter conservation is clearly observed.

\section{CONCLUSIONS}

We recently reported a different phase transition scenario in the context of a planar Ising system with a grain boundary [1]. In this GZZ transition, when the system is below the transition point and in a zigzag phase, an interesting relaxation process which confines matter transport into the grain boundary was revealed.

In this paper we have studied this relaxation dynamics with the dynamical confinement in detail. First, by performing continuous time MC simulations with local Kawasaki dynamics, and second by deriving Langevin equations for the relevant HSOS model. The Ising simulations establish two observations, the system with a grain boundary relaxes transporting matter mostly through the boundary, via the

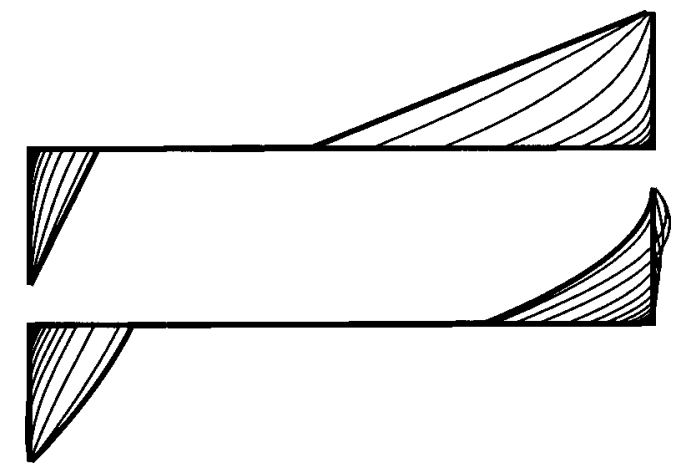

FIG. 13. The relaxation of the inhomogeneous systems $\tau_{1}>\tau_{2}$ $>b \tau_{0}$. The one above is the solution to Eq. (11), i.e., without matter conservation and the one below to Eq. (34), i.e., with conservation. The thin lines show the interface at intermediate times. The system with matter conservation cannot reach the grand canonical equilibrium and the interface sections bend in accordance with the variational calculations carried out in the text.

minimum energy paths, whereas the system without a grain boundary uses capillary fluctuations to equilibrate. The absence of the capillary fluctuation in the middle interface section sitting at the grain boundary combined with the $K_{2}$ bond strength independence of the phase boundary led us to formulate the problem in terms of the HSOS model in Sec. IV. This formulation made it possible to derive Langevin dynamics for the model system. We derived and solved the corresponding Langevin equations with and without matter conservation in Sec. V. While the HSOS model and the derived dynamics give a good description of the relaxation process for a system with a grain boundary, it is important to notice that once the grain boundary is removed, the system relaxes using capillary fluctuations and hence the HSOS picture is not valid anymore. Finally, in the end of Sec. V we demonstrated how the introduction of inhomogeneity in the system, i.e., the half systems have different couplings $\tau_{i}$, leads to different equilibrium configurations depending whether or not matter conservation is imposed.

\section{ACKNOWLEDGMENTS}

All the authors thank Professor K. Kaski for hospitality in Helsinki. D.B.A. thanks the Laboratory of Computational Engineering at the Helsinki University of Technology (LCEHUT) for appointing him to a Visiting Professorship. D.B.A. and A.J.W. acknowledge financial support from the EPSRC under Grant Nos. GR/M04426 and GR/R83712/01, respectively. V.M. was partially supported by the Academy of Finland, Research Centre for Computational Science and Engineering, Project No. 44897 (Finnish Centre of Excellence Programme 2000-2005). Computing time made available by collaboration of LCE-HUT and Wolfson College (Oxford) is greatly appreciated.

\section{APPENDIX: GAUSSIAN HSOS}

We present here, for completeness, the calculation to derive the static configuration for a SOS model under the 
Gaussian approximation (8). This approximation will only be valid in the static case for systems with $\theta$ small. Nevertheless, the calculation is simple and follows precisely the methodology for the system defined by Eq. (7).

The partition function is defined as

$$
Z=\int_{-\infty}^{\infty} d h_{0} \cdots d h_{N} \prod_{i=1}^{N} e^{-(K / 2)\left(h_{i}-h_{i-1}\right)^{2}} e^{-K b\left|h_{n}-n\right|} \delta\left(h_{0}\right) .
$$

We can form the intermediate integrations as an elementary transfer problem in an analogous way to that found in Ref. [20]. The only difference here is the change of transfer kernel; here we use

$$
\langle x|T| y\rangle=e^{-K / 2(x-y)^{2}}
$$

and thus the transfer problem is defined by

$$
\int_{-\infty}^{\infty}\langle x|T| y\rangle \psi_{q}(y) d y=\lambda^{(q)} \psi_{q}(x)
$$

The $\psi_{q}(x)$ form a complete set of eigenfunctions with the usual orthogonality relations. In this problem we are only interested in the scattering states $e^{i k x}$, and these have eigenvalues

$$
\lambda^{(q)}=\sqrt{\frac{2 \pi}{K}} e^{\left(k^{2} / 2 K\right)} .
$$

As in Ref. [20] we select only the even eigenfunctions, which also contain some unimportant normalization constants. As might be expected from the simple Gaussian form of Eq. (A4) the subsequent integrals can be performed exactly. Inserting the transfer problem into the form for the partition function and performing the trivial $h_{0}$ integration yields

$$
Z=\int_{-\infty}^{\infty} d h_{N} e^{-K b\left|h_{n}-n\right|}\left(\frac{2 \pi}{K}\right)^{N / 2} \frac{2 \pi K}{N} e^{-h_{N}^{2} K / 2 N} .
$$

This expression can be split and then recast in the form of complementary error functions. We find

$$
\begin{aligned}
Z= & \left(\frac{2 \pi}{K}\right)^{N / 2} e^{K b^{2} N / 2}\left[e^{-K b N \tan \phi} \operatorname{erfc}\left(\sqrt{\frac{K N}{2}}(b-\tan \phi)\right)\right. \\
& \left.+e^{K b N \tan \phi} \operatorname{erfc}\left(\sqrt{\frac{K N}{2}}(b+\tan \phi)\right)\right] .
\end{aligned}
$$

It should be immediately clear that in the $N$ large limit the second of these error functions will not contribute (which, as it emerges from excursions of the interface beyond the angular minima, should not be surprising). The dominant behavior of the former error function is controlled by the sign of $b-\tan \phi$. We find

$$
f=\lim _{N \rightarrow \infty} \frac{1}{N} \ln Z=\frac{1}{2} \ln \frac{2 \pi}{K}+\left\{\begin{array}{cc}
\frac{K b^{2}}{2}-K b \tan \phi & b<\tan \phi \\
\frac{K \tan ^{2} \phi}{2} & b>\tan \phi
\end{array}\right.
$$

for the energy and this implies that the order parameter for this model, $\left|h_{N}-n\right|$, can be computed as

$$
\left\langle\left|h_{N}-n\right|\right\rangle \equiv-\frac{1}{K} \frac{\partial}{\partial b} \ln Z=\left\{\begin{array}{cl}
K(b-\tan \phi) & b<\tan \phi \\
0 & b>\tan \phi
\end{array},\right.
$$

where we can clearly see its continuous divergence.

We remark here also that we should not be surprised by the seemingly anomalous result for the phase boundary. As we have explicitly treated the center tier differently the model will not respect the limit $b \rightarrow 1$.
[1] D. B. Abraham, V. Mustonen, and A. J. Wood, Phys. Rev. Lett. 93, 076101 (2004).

[2] D. B. Abraham, in Phase Transitions and Critical Phenomena-Volume 10, edited by C. Domb and J. L. Lebowitz (Academic, London, 1988).

[3] S. Dietrich, in Phase Transitions and Critical PhenomenaVolume 12, edited by C. Domb and J. L. Lebowitz (Academic, London, 1988).

[4] D. B. Abraham, Phys. Rev. Lett. 44, 1165 (1980).

[5] H. N. V. Temperley, Proc. Cambridge Philos. Soc. 48, 683 (1952).

[6] S. T. Chui and J. D. Weeks, Phys. Rev. B 23, 2438 (1981).

[7] J. T. Chalker, J. Phys. A 14, 2431 (1981).

[8] T. W. Burkardt, J. Phys. A 14, L63 (1981).

[9] J. A. Nieminen, D. B. Abraham, M. Karttunen, and K. Kaski, Phys. Rev. Lett. 69, 124 (1992).

[10] J. A. Nieminen and T. Ala-Nissila, Phys. Rev. E 49, 4428 (1994).
[11] J. De Coninck, U. D’Ortona, J. Koplik, and J. R. Banavar, Phys. Rev. Lett. 74, 928 (1995).

[12] D. B. Abraham, R. Cuerno, and E. Moro, Phys. Rev. Lett. 88, 206101 (2002).

[13] A. L. Barabási and H. E. Stanley, Fractal Concepts in Surface Growth (Cambridge University Press, Cambridge, England, 1995).

[14] M. N. Popescu and S. Dietrich, Phys. Rev. E 69, 061602 (2004).

[15] M. Kac and J. Logan, in Studies in Statistical Mechanics VII, edited by E. W. Montroll and J. L. Lebowitz (Academic, New York, 1983).

[16] D. B. Abraham, F. T. Latrémolière, and P. J. Upton, Phys. Rev. Lett. 71, 404 (1993).

[17] D. B. Abraham, P. Collet, J. De Coninck, and F. Dunlop, J. Stat. Phys. 61, 509 (1990).

[18] D. B. Abraham and D. A. Huse, Phys. Rev. B 38, 7169 (1988).

[19] P. Collet, F. Dunlop, and T. Gobron, J. Stat. Phys. 79, 215 
(1995).

[20] D. B. Abraham, V. Mustonen, and A. J. Wood, J. Phys. A 37, L233 (2004).

[21] M. E. Fisher, J. Stat. Phys. 34, 667 (1984).

[22] F. Wang and D. P. Landau, Phys. Rev. Lett. 86, 2050 (2001).

[23] B. J. Schulz, K. Binder, and M. Müller, Interface (USA) 13, 477 (2002).

[24] D. B. Abraham, V. Mustonen, and A. J. Wood, Europhys. Lett. 63, 408 (2003).

[25] D. B. Abraham, V. Mustonen, and A. J. Wood (unpublished).
[26] B. Schmittmann and R. K. P. Zia, in Phase Transitions and Citical Phenomena-Volume 17, edited by C. Domb and J. L. Lebowitz (Academic, London, 1995).

[27] M. E. J. Newman and G. T. Barkema, Monte Carlo Methods in Statistical Physics (Oxford University Press, New York, 1999).

[28] D. B. Abraham and P. J. Upton, Phys. Rev. B 37, 3835 (1988).

[29] R. Bausch, V. Dohm, H. K. Janssen, and R. K. P. Zia, Phys. Rev. Lett. 47, 1837 (1981).

[30] J. Zinn-Justin, Quantum Field Theory and Critical Phenomena (Oxford University Press, Oxford, 1993). 